\title{
Psychisches Befinden in der Bevölkerung während der COVID-19-Pandemie
}

\author{
Mental Health Status in the Community During the \\ COVID-19-Pandemic
}

Autoren

Christine Kuehner, Katharina Schultz, Peter Gass, Andreas Meyer-Lindenberg, Harald Dreßing

Institut

Abteilung Psychiatrie und Psychotherapie, Zentralinstitut

für Seelische Gesundheit, Mannheim, Medizinische Fakultät

Mannheim der Universität Heidelberg

Schlüsselwörter

COVID-19, Bevölkerungsstichprobe, psychische

Gesundheit, Risikofaktoren, Resilienz

Keywords

COVID-19, population based sample, mental health, risk

factors, resilience

Online-Publikation 21.8.2020

Bibliografie

Psychiat Prax 2020; 47: 361-369

DOI 10.1055/a-1222-9067

ISSN 0303-4259

(C) 2020. Thieme. All rights reserved.

Georg Thieme Verlag KG, Rüdigerstraße 14,

70469 Stuttgart, Germany

Korrespondenzadresse

Prof. Dr. Christine Kühner, Dipl.-Psych., Arbeitsgruppe

Verlaufs- und Interventionsforschung, Abteilung Psychiatrie und Psychotherapie, Zentralinstitut für Seelische Gesundheit, Mannheim, J 5, 68159 Mannheim, Deutschland

Christine.Kuehner@zi-mannheim.de

Zusätzliches Material finden Sie unter:

https://doi.org/10.1055/a-1222-9067

\section{ZUSAMMENFASSUNG}

Ziel der Studie Zusammenhangsanalyse von wahrgenommenen Alltagsbelastungen während der COVID-19-Krise und Persönlichkeitsaspekten mit dem aktuellen psychischen Befinden in der Bevölkerung sowie Vergleich der psychischen Symptomatik mit einem Survey von 2018.
Methodik Zufallsstichprobe aus der Bevölkerung $(n=721)$, die zu coronabezogenen Einschätzungen, Persönlichkeitsaspekten und aktuellem psychischen Befinden (WHO-5, PHQ-D) schriftlich befragt wurde.

Ergebnisse Angst um die Gesundheit Nahestehender, Belastungen durch Ausgangsbeschränkungen und vermehrter Substanzkonsum sowie psychische Risiko- und Resilienzfaktoren trugen im statistischen Modell unabhängig zur Vorhersage des psychischen Befindens bei. Ausprägungen und Häufigkeiten psychischer Symptomatik unterschieden sich nicht zwischen 2018 und 2020.

Schlussfolgerung Dies ist eine erste Studie in Deutschland, die Ergebnisse aus einer bevölkerungsbezogenen Zufallsstichprobe zu Determinanten psychischen Befindens während der COVID-19-Krise und einen Vergleich der psychischen Symptomatik mit einer präpandemischen Referenzstichprobe vorlegt.

\section{ABSTRACT}

Objective Analysis of associations between perceived daily life strain during the COVID-19-crisis and personality aspects with current psychological wellbeing in the general population and comparison of mental health indicators with those from a previous survey in 2018.

Methods Written survey in a random sample from the general population $(n=721)$ on Corona-related assessments, personality aspects and current mental health (WHO-5, PHQ-D).

Results Fear for the health of close persons, strain regarding going out restrictions, increased substance use and psychological risk- and resilience factors were independently related to current mental health. Extent and frequencies of mental health symptomatology did not differ between 2018 and 2020.

Conclusion This is a first study in Germany reporting results from a population-based random sample on determinants of mental health during the COVID-19-crisis and a comparison of mental health symptomatology with prepandemic data from the same population. 


\section{Einleitung}

Die COVID-19-Pandemie hat sich inzwischen in mehr als 180 Ländern ausgebreitet, weltweit wurden bislang mehr als 12 Millionen Menschen infiziert und über 550000 sind im Zusammenhang mit COVID-19 gestorben (Stand: 13.7.2020) [1]. Deutschland zählte ab Mitte März 2020 zu den stark betroffenen Ländern Europas. Es wurden zum Teil weitreichende Maßnahmen erlassen, um die Ausbreitung der Pandemie einzudämmen. Der anhaltende weitgehende Stillstand des öffentlichen Lebens mit Schließung von Bildungseinrichtungen und Kinderbetreuungsstätten, gravierenden Einschnitten auf dem Arbeitsmarkt, Kontaktverboten und anderen Beschränkungen des täglichen Lebens, die erst seit Mai 2020 wieder schrittweise gelockert werden, stellt die Menschen vor große psychische Herausforderungen. Hinzu kommt die anhaltende Unsicherheit über die längerfristige Entwicklung und weitere wirtschaftliche und soziale Folgen der Pandemie.

Es stellt sich die Frage, wie sich die COVID-19-Pandemie auf die psychische Gesundheit in der Allgemeinbevölkerung auswirkt. Bisherige Reviews befassen sich zumeist mit den psychischen Auswirkungen früherer Infektionskrisen [2, 3] oder mit aktuellen Auswirkungen der Corona-Krise für bestimmte Gruppen wie medizinisches Personal [4-6]. Studien zu psychischen Auswirkungen in der Allgemeinbevölkerung liegen bislang dagegen nur vereinzelt vor [6].

Ein Review über 24 Studien aus früheren Infektionspandemien [2] berichtet negative Effekte von Quarantänemaßnahmen, die u.a. posttraumatische, depressive und Angstsymptome sowie Gereiztheit und Ärger beinhalten. Als Stressoren werden längere Quarantänedauer, Infektionsängste, Frustration, Langeweile, unzureichende Informationen, finanzielle Einbußen und wahrgenommenes Stigma genannt. Einige, jedoch nicht alle der überblickten Studien fanden länger dauernde Effekte auf die psychische Gesundheit. Ein vergleichbarer Review [3] über 13 Studien beschreibt ähnliche psychische Folgen und schlussfolgert, dass Tätige im Gesundheitswesen eine besonders vulnerable Gruppe darstellen.

Aktuelle Reviews zu Auswirkungen der COVID-19-Pandemie auf das Personal im Gesundheitswesen [4-6] zeigen ebenfalls erhebliche psychische Belastungen, wobei die Angaben zur Prävalenz psychischer Symptomatik in den Reviews trotz des weitgehenden Einschlusses derselben Studien stark variieren. Als Mediatoren werden Art des Personalmanagements, präventive Interventionen sowie Resilienz und soziale Unterstützung diskutiert [4]. Die erhöhte psychische Belastung von im Gesundheitswesen arbeitenden Menschen während der COVID-19Pandemie hat bereits zur Entwicklung und Evaluation von spezifischen Präventionsprogrammen geführt [7].

Der Review von Vindegaard u. Benros [6] zu psychischen Auswirkungen der COVID-19-Pandemie in der Allgemeinbevölkerung umfasst 19 Studien mit sehr heterogenen Studienpopulationen, die überwiegend in China $(n=14)$ durchgeführt wurden, aus Europa sind 4 Studien enthalten. Die Mehrzahl der Studien $(n=12)$ nutzte Online-Surveys. Auch hier resultiert eine große Variabilität in der Ausprägung psychischer Beschwerden. Allgemeine Risikofaktoren waren weibliches Geschlecht (je- doch nicht in allen Studien), jüngeres Alter, niedriges Ausbildungsniveau oder niedriges Einkommen, bereits bestehende psychische und körperliche Erkrankungen sowie schlechte selbsteingeschätzte Gesundheit. Daneben waren jedoch auch coronaspezifische Belastungsfaktoren und Verhaltensweisen mit ausgeprägter psychischer Symptomatik assoziiert, wie geringe Informiertheit über das Virus, Einschränkungen des Alltaglebens, aktuelle Isolation, Infektionen bei nahestehenden Personen, instabile ökonomische Verhältnisse und stärkere Nutzung sozialer Medien während der Pandemie [6]. Lediglich eine der Studien [8] verglich das psychische Befinden vor (2016) und während der Pandemie (2020) aus 2 landesweiten dänischen Online-Surveys, sie fand eine Verschlechterung der Depressionswerte, gemessen mit dem WHO-5-Fragebogen [9, 10], um durchschnittlich 2,3 Punkte sowie eine Erhöhung des Anteils auffälliger WHO-5-Skalenwerte von 22,5 auf 25,4\%.

Bezüglich der Methodik bisheriger Studien aus der Allgemeinbevölkerung wird kritisiert, dass in der Regel Online-Befragungen ohne Zufallsziehungen von Stichproben durchgeführt werden, häufig kombiniert mit Schneeballverfahren über verschiedene soziale Netzwerke, was die Repräsentativität zusätzlich einschränkt [11]. Jedoch erlauben nur populationsbasierte Zufallsziehungen Aussagen zu Nonrespondern und eine valide Anwendung statistischer Verfahren. Mit einer Ausnahme [8] fehlen zudem Studien, die mit identischen Instrumenten in denselben Studienpopulationen die psychische Symptomatik außerhalb und während der Pandemie untersuchten.

Bislang liegen auch in Deutschland noch keine Studien zum psychischen Befinden während der COVID-19-Krise vor, die auf bevölkerungsbezogenen Zufallsstichproben beruhen und zeitnahe Vergleichswerte für die Häufigkeit psychischer Störungen in Referenzpopulationen außerhalb der Pandemie liefern. Zudem fehlen detaillierte Analysen zum Zusammenhang zwischen wahrgenommenen coronabezogenen Belastungen und Verhaltensänderungen im Alltag (z. B. veränderter Substanzkonsum) und psychischem Befinden. Schließlich wurde der Einfluss psychischer Risiko- und Resilienzfaktoren im Kontext der COVID-19-Pandemie bislang nur unzureichend untersucht. Persönlichkeitsfaktoren wie emotionale Stabilität, Gewissenhaftigkeit, Optimismus, Pessimismus, Kontrollüberzeugungen und Grübelneigung, die generell mit psychischer Gesundheit assoziiert sind (vgl. [12-15]), dürften auch das aktuelle psychische Befinden und die Wahrnehmung coronabezogener Alltagsbelastungen beeinflussen.

Am Zentralinstitut für Seelische Gesundheit, Mannheim, führten wir im Zeitraum vom 24.4.-23.5.2020 einen schriftlichen Survey im Kontext der COVID-19-Pandemie an einer Zufallsstichprobe der Mannheimer Bevölkerung durch. Ziele der vorliegenden Studie sind

- Zusammenhänge von soziodemografischen Merkmalen, coronabezogenen Einschätzungen und psychischen Risikound Resilienzfaktoren mit dem aktuellen psychischen Befinden zu untersuchen sowie

- das aktuelle psychische Befinden einschließlich quantitativer Syndromwerte und -diagnosen psychischer Störungen mit Daten eines früheren Surveys in derselben Population aus dem Jahr 2018 zu vergleichen. 


\section{Methode}

\section{Studiendesign}

Im April 2020 wurden per Zufall aus der Einwohnermeldedatei der Stadt Mannheim die Adressen von jeweils 1000 Frauen und 1000 Männern im Alter von 18-65 Jahren mit deutscher Staatsangehörigkeit gezogen. Diesen Personen wurde ein Fragebogen zu aktuellen coronabezogenen Einschätzungen zusammen mit Kurzfragebögen zu Persönlichkeitsaspekten sowie dem WHO-5-Well-Being-Index (WHO-5, s.u.) und dem Gesundheitsfragebogen für Patienten (PHQ-D, s.u.) in KW 17 zugeschickt mit der Bitte, die ausgefüllten Unterlagen in einem beiliegenden frankierten Rückumschlag anonym zurückzusenden. 14 Tage später erfolgte ein einmaliges Erinnerungsschreiben an alle Adressen. Im Jahr 2018 waren im Rahmen eines Bevölkerungssurveys in Mannheim, dort im Kontext einer Umfrage zum Thema Stalking, mit identischem Studiendesign dieselben Instrumente zur Erfassung des psychischen Befindens vorgegeben worden [16].

\section{Instrumente}

Fragebogen zu aktuellen Auswirkungen der Coronakrise. Der eingesetzte Kurzfragebogen enthält neben soziodemografischen Informationen 9 Fragen zur subjektiven Informiertheit über das Corona-Virus, zur Einschätzung der Angebrachtheit von Maßnahmen zur Eindämmung des Virus sowie zur Angst um die eigene Gesundheit, Angst um die Gesundheit nahestehender Personen, Belastung durch Ausgangsbeschränkungen, Sorgen um Arbeitsplatz und Finanzen, Auswirkungen auf die familiäre Situation sowie zu vermehrtem Alkohol- und sonstigem Substanzkonsum während der Corona-Krise. Das Antwortformat ist auf 5-Punkt-Likertskalen vorgegeben ( $0=$ überhaupt nicht, 1 = eher nicht, 2 = teils/teil, 3 = eher ja, 4 = sehr; Ausnahme Maßnahmen: $0=$ deutlich zu wenig streng, $1=$ eher zu wenig streng, 2 =genau richtig, $3=$ eher zu streng, $4=$ deutlich zu streng) ${ }^{1}$.

Big Five Inventory-10 (BFI-10, [13]). Das BFI-10 ist auf der Basis des 5-Faktoren-Modells der Persönlichkeit konstruiert. Jeweils 2 Items erfassen anhand 5-stufiger Likert-Skalen die abstrakten Dimensionen Extraversion, Verträglichkeit, Gewissenhaftigkeit, Neurotizismus und Offenheit. Der Fragebogen ist für den Einsatz in größeren Stichproben der Allgemeinbevölkerung konstruiert und besitzt gute faktorielle, konvergente und Konstruktvalidität [13].

Life Orientation Test (LOT-R, [14]). Der LOT-R erfasst die Konstrukte dispositioneller Optimismus und dispositioneller Pessimismus anhand von jeweils 3 Items. Die 2-dimensionale Struktur des Fragebogens wurde aufgrund vielfältiger Befunde bestätigt [14]. Der LOT-R zeigt gute psychometrische Kennwerte, ist international weit verbreitet und wird für den Einsatz in epidemiologischen Studien empfohlen [14].

Internale-Externale-Kontrollüberzeugung-4 (IE-4, [12]). Der IE-4 enthält 4 Items, von denen jeweils 2 Items das Ausmaß internaler und externaler Kontrollüberzeugung erfassen. Die psychometrische Überprüfung an großen Stichproben beschei- nigt dem Kurzfragebogen gute Reliabilitäts- und Validitätskennwerte [12].

Grübelneigung. Die selbsteingeschätzte Grübeltendenz wurde mit dem Item „Ich neige zum Grübeln und kann mich schlecht davon lösen“ erfasst (in Analogie zu Studien, die Grübeln im Kontext von Alltagserleben untersuchen (z. B. [17]).

Die Items aus BFI-10, LOT-R, IE-4 und Grübelneigung werden auf 5-stufigen Likert-Skalen eingeschätzt.

WHO-5-Well-Being-Index (WHO-5, [9, 10]). Der WHO-5 ist ein Fragebogen zur Einschätzung des psychischen Befindens. Der Fragebogen besteht aus 5 positiv formulierten Items, die auf 6-stufigen Likert-Skalen eingeschätzt werden ( 5 = die ganze Zeit, 4 =meistens, 3 = etwas mehr als die Hälfte der Zeit, 2 = etwas weniger als die Hälfte der Zeit, $1=a b$ und zu, $0=$ zu keinem Zeitpunkt). Der Summenwert (0-25) wird mit 4 multipliziert, damit liegt der Range zwischen 0 und 100.Der WHO-5 wurde inzwischen in über 30 Sprachen übersetzt und psychometrisch umfangreich getestet. Rasch-Analysen bestätigen die Eindimensionalität der Skala, zu der jedes Einzelitem einen unabhängigen Beitrag leistet [10]. Der Fragebogen wurde inzwischen weltweit in unterschiedlichen epidemiologischen und klinischen Settings als generisches Wohlbefindlichkeitsmaß eingesetzt [10], darüber hinaus ist der WHO-5 ein sensitives und spezifisches Screeninginstrument für Depression [9, 10]. Ein Wert von <50 stellt eine Indikation für eine eingehendere Untersuchung hinsichtlich eines depressiven Syndroms dar [10]. In der vorliegenden Studie diente der WHO-5 in den Analysen zu Zusammenhängen zwischen Soziodemografie, coronabezogenen Einschätzungen und psychischem Befinden als zentrales Befindlichkeitsmaß, da er weniger spezifische Symptome abfragt als die einzelnen PHQ-D-Syndromskalen (s.u.).

Gesundheitsfragebogen für Patienten (PHQ-D, [18]). Der PHQ-D ermöglicht die Berechnung von Syndromdiagnosen nach DSM-IV für die häufigsten psychischen Störungen (somatoformes Syndrom, depressive Syndrome, Angstsyndrome, Verdacht auf Essstörungen und Alkoholmissbrauch). Neben der kategorialen Auswertung nach DSM-IV lassen sich quantitative Syndromscores für Angstsymptomatik, Depressionssymptomatik, somatoforme Symptomatik und Stresswerte berechnen. Internationale Studien bescheinigen dem PHQ-D sehr gute Reliabilitäts- und diagnostische Validitätswerte [18]. Neben dem WHO-5 wurde der PHQ-D in der vorliegenden Studie zum Vergleich des Vorliegens einer oder mehrerer Syndromdiagnosen und von quantitativen Syndromscores mit dem 2018er-Survey eingesetzt. Das Vorliegen einer Syndromdiagnose war erfüllt, wenn die Kriterien für mindestens eine der oben aufgeführten Syndrome (somatoformes Syndrom, depressive Syndrome, Angstsyndrome, Verdacht auf Essstörungen und Alkoholmissbrauch) im PHQ-D erfüllt waren, für eine Komorbiditätsdiagnose die Kriterien für mindestens 2 Syndrome. Im 2020er-Survey betrugen die Pearson-Korrelationen zwischen WHO-5-Summenwert und den Syndromscores des PHQ-D r = - 0,697 (PHQD Depression), $r=-0,598$ (PHQ-D Angst), $r=-0,499$ (PHQ-D somatoforme Symptome) und $r=-0,558$ (PHQ-D Stresswerte, alle ps. $<0,001)$.

\footnotetext{
${ }^{1}$ Die Fragen sind auf Anfrage bei der Erstautorin erhältlich.
} 


\section{Statistische Analysen}

Zur statistischen Analyse der Daten diente das Programm IBM Statistics, SPSS 25. Einfache Berechnungen zur Repräsentativität der COVID-19-Umfragestichprobe erfolgten durch einen Vergleich entsprechender Alters- und Geschlechtsverteilungen mit der Bevölkerungsstatistik der Stadt Mannheim anhand von Pearson's Chi²-Tests. Die Verteilung von soziodemografischen Merkmalen und Angaben zu aktuellen Auswirkungen der Corona-Krise werden in deskriptiven Analysen in Form von Prozentwerten dargestellt. Zur Prüfung des Zusammenhangs zwischen Soziodemografie, coronabezogenen Einschätzungen und Persönlichkeitsfaktoren mit dem psychischen Befinden (WHO-5Summenwert) wurden aufeinanderfolgend 3 Analysen mit dem allgemeinen linearen Modell (ALM) berechnet: Modell 1 enthält nur die soziodemografischen Variablen Alter, Geschlecht und Bildungsstand (Skalenwerte $0=$ Hauptschulabschluss bis 6 =Fachhochschul-/Hochschulabschluss), Modell 2 die Hinzunahme der coronabezogenen Einschätzungen, und Modell 3 die weitere Aufnahme der persönlichkeitsassoziierten Merkmale. Dargestellt werden neben der Signifikanz des jeweiligen Gesamtmodells Regressionskoeffizienten (B), Standardfehler (SE) und Signifikanzniveaus der unabhängigen Variablen. Einfache Vergleiche zur Stichprobenzusammensetzung der Umfragen 2020 und 2018 erfolgten mit Pearson's Chi²-Tests und mit t-Tests. Alle Analysen zum Vergleich quantitativer und kategorialer psychischer Symptommaße von 2020 mit 2018 wurden unter Kontrolle von Geschlecht, Alter und Bildungsgrad durchgeführt (WHO-5-Summenwert mit ALM, quantitative Syndromscores des PHQ-D anhand einer multivariaten Kovarianzanalyse, WHO-5 Cut-off $<50$ [0/1] und Vorliegen mindestens einer bzw. mehrerer Diagnosen nach PHQ [0/1] anhand von binären logistischen Regressionen). Sämtlichen Analysen wurde ein Signifikanzniveau von $\alpha=0,05$ zugrunde gelegt.

\section{Ergebnisse}

\section{Responserate und Repräsentativität der Umfragen 2020 und 2018}

Die Versendung der Fragebögen für die COVID-19-Umfrage erfolgte am Ende von KW 17. Die vorliegenden Auswertungen beziehen sich auf die nachfolgenden Rückläufe des 4-wöchigen Zeitraums von KW 18-21 (24.4.-23.5.). Von den 2000 versendeten Fragebögen waren 1960 an gültige Adressen zustellbar, der Rücklauf betrug bis einschließlich KW 21 mit 721 auswertbaren Fragebögen somit 36,9\%.

Unter Bildung von 5 Altersklassen war die Altersverteilung der aktuellen Stichprobe nicht signifikant von der Altersverteilung der Mannheimer Bevölkerung mit deutscher Staatsangehörigkeit im Alter zwischen 18 und 65 Jahren verschieden $\left(\mathrm{Chi}^{2}=4,64\right.$ [4]; $\left.\mathrm{p}=0,326\right)$. Allerdings war der Frauenanteil mit $57,1 \%$ gegenüber der Mannheimer Bevölkerung in diesem Altersbereich (18-65; 49,7\%) signifikant erhöht $\left(\mathrm{Chi}^{2}=15,7\right.$ [1]; $p<0,001$ ). Die Umfrage 2018 hatte dagegen mit 444 Respondern von 1930 zustellbaren Fragebögen nur eine Responserate von $23,0 \%$ und lag damit signifikant niedriger $\left(\mathrm{Chi}^{2}=88,0\right.$ $[1] ; p<0,001)$. Bezüglich Altersverteilung $(t[1144]=1,11 ; p=$
$0,296)$ und Bildungsgrad $(t[1139]=-0,22 ; p=0,826)$ waren beide Stichproben vergleichbar, jedoch hatten 2018 mehr Frauen teilgenommen (64,1 vs. 57,1\%; $\mathrm{Chi}^{2}=5,44$ [1]; $\mathrm{p}=0,002$ ).

\section{Stichprobencharakteristika der COVID-19-Umfrage 2020}

Das durchschnittliche Alter der Umfrageteilnehmenden lag bei 41,8 Jahren $(S D=14,2), 57,1 \%$ waren weiblich. Insgesamt $12,1 \%$ hatten Hauptschulabschluss, 21,0\% Realschulabschluss oder gleichwertig, 22,1\% Fachhochschul-/Hochschulreife und 44,4\% einen Fachhochschul-/Hochschulabschluss. Ledig waren $47,1 \% ; 43,7 \%$ verheiratet und 9,2\% verwitwet, geschieden oder getrennt lebend. Aktuell lebten $66,3 \%$ mit einem Partner oder einer Partnerin zusammen. Bei 21,8\% der Stichprobe lebte mindestens ein Kind unter 18 Jahren im eigenen Haushalt.

\section{Deskriptive Analysen zu coronabezogenen Einschätzungen in der Bevölkerung}

> Online-Abb. 1 zeigt die Verteilung der Einschätzungen zu 8 der 9 erfragten coronabezogenen Bereiche. Demnach fühlten sich nur 5,5\% der Befragten über das Corona-Virus nicht oder eher nicht ausreichend informiert. Angst um die eigene Gesundheit (alle nachfolgenden Angaben teils/teils bis sehr) machten sich 33,7\%, um die Gesundheit nahestehender Personen dagegen $82,6 \%$. Durch die Ausgangsbeschränkungen belastet fühlten sich $62,7 \%, 35,8 \%$ machten sich aufgrund der Krise Sorgen um ihren Arbeitsplatz oder um finanzielle Einbußen und für $65,0 \%$ hatte die Krise Auswirkungen auf die aktuelle familiäre Situation, z. B. durch die häusliche Betreuung der Kinder oder vermehrte/verminderte Kontakte zu anderen Angehörigen. Zum Alkoholkonsum gaben 19,1\% an, diesen während der Corona-Krise erhöht zu haben, bezüglich sonstiger psychotroper Substanzen einschließlich Medikamente lag der entsprechende Anteil bei 8,5\%.

Die Maßnahmen zur Eindämmung der Corona-Krise in Deutschland hielten 51,7\% für genau richtig, 27,0 \% für eher/ deutlich zu wenig streng und 21,3\% für eher/deutlich zu streng.

Zusammenhänge von soziodemografischen Merkmalen, coronabezogenen Einschätzungen und Persönlichkeitsmerkmalen mit dem aktuellen psychischen Befinden In Modell 1 ( $\bullet$ Tab. 1) zeigen sich signifikante Zusammenhänge von Alter und Bildungsniveau mit dem WHO-5-Summenwert. Ältere Personen $(p=0,003)$ und Personen mit höherem Bildungsniveau $(p=0,040)$ berichten höheres Wohlbefinden, während mit dem Geschlecht kein Zusammenhang besteht. Das Gesamtmodell trägt signifikant zur Vorhersage der WHO-5-Werte bei $(\mathrm{F}[3,698]=4,20 ; \mathrm{p}<0,006)$ und erklärt $14 \%$ der Variation der Befindlichkeitswerte (korrigiert). Unter Einschluss der coronabezogenen Einschätzungen (Modell 2, \Tab.1) erhöht sich die erklärte Varianz auf 22\% (korrigiert, Signifikanz des Gesamtmodells: $F[13,641]=15,3 ; p<0,001)$. Bei gleichzeitiger Berücksichtigung der soziodemografischen Merkmale sind ein wahrgenommener niedriger Informationsgrad über das Corona-Virus $(p<0,001)$, höhere Ängste um die eigene Gesundheit $(p=0,038)$ und um die Gesundheit Nahestehender $(p=0,042)$, höhere Belastungen durch Ausgangsbeschränkungen $(p<$ 0,001), Sorgen um den Arbeitsplatz und/oder finanzielle Ein- 
- Tab. 1 Zusammenhänge von soziodemografischen Merkmalen, coronabezogenen Einschätzungen und Persönlichkeitsmerkmalen mit dem aktuellen psychischen Befinden (WHO-5).

\begin{tabular}{|c|c|c|c|c|c|c|c|c|c|}
\hline & \multicolumn{3}{|c|}{ Modell $1(n=702)$} & \multicolumn{3}{|c|}{ Modell $2(n=655)$} & \multicolumn{3}{|c|}{ Modell $3(n=651)$} \\
\hline & $B^{6}$ & $\mathrm{SE}^{7}$ & $\mathbf{p}$ & $B^{6}$ & $\mathrm{SE}^{7}$ & $\mathbf{p}$ & $B^{6}$ & $\mathrm{SE}^{7}$ & p \\
\hline \multicolumn{10}{|l|}{ Soziodemografie } \\
\hline Geschlecht ${ }^{1}(n=718)$ & 0,435 & 0,426 & 0,307 & 0,175 & 0,397 & 0,659 & 0,256 & 0,377 & 0,498 \\
\hline Alter $(n=719)$ & 0,046 & 0,015 & 0,003 & 0,025 & 0,015 & 0,099 & 0,007 & 0,014 & 0,500 \\
\hline Bildungsniveau $(n=711)$ & 0,212 & 0,103 & 0,040 & 0,091 & 0,100 & 0,363 & 0,010 & 0,090 & 0,910 \\
\hline \multicolumn{10}{|l|}{ coronabezogene Einschätzungen } \\
\hline ausreichend informiert $(n=694)$ & & & & 0,914 & 0,234 & $<0,001$ & 0,384 & 0,208 & 0,066 \\
\hline $\begin{array}{l}\text { Maßnahmen }{ }^{2}(\mathrm{n}=686) \\
1=\text { zu wenig streng } \\
2 \text { = zu streng }\end{array}$ & & & & $\begin{array}{l}-0,039 \\
-0,319\end{array}$ & $\begin{array}{l}0,471 \\
0,581\end{array}$ & $\begin{array}{l}0,933 \\
0,583\end{array}$ & $\begin{array}{l}-0,344 \\
0,289\end{array}$ & $\begin{array}{l}0,512 \\
0,416\end{array}$ & $\begin{array}{l}0,501 \\
0,488\end{array}$ \\
\hline $\begin{array}{l}\text { Angst um eigene Gesundheit } \\
(n=696)\end{array}$ & & & & $-0,489$ & 0,236 & 0,038 & $-0,015$ & 0,212 & 0,944 \\
\hline $\begin{array}{l}\text { Angst um Gesundheit Nahestehender } \\
(n=694)\end{array}$ & & & & $-0,449$ & 0,220 & 0,042 & $-0,389$ & 0,193 & 0,044 \\
\hline $\begin{array}{l}\text { Belastung durch Ausgangs- } \\
\text { beschränkung }(n=694)\end{array}$ & & & & $-1,128$ & 0,190 & $<0,001$ & $-0,939$ & 0,167 & $<0,001$ \\
\hline Sorgen um Arbeit/Finanzen $(n=694)$ & & & & $-0,349$ & 0,162 & 0,031 & $-0,100$ & 0,144 & 0,485 \\
\hline $\begin{array}{l}\text { Auswirkungen auf familiäre } \\
\text { Situation }(n=698)\end{array}$ & & & & $-0,192$ & 0,149 & 0,199 & $-0,233$ & 0,131 & 0,076 \\
\hline mehr Alkohol $(n=697)$ & & & & 0,067 & 0,186 & 0,716 & 0,023 & 0,163 & 0,888 \\
\hline mehr sonstige Substanzen $(n=697)$ & & & & $-1,462$ & 0,261 & $<0,001$ & $-1,091$ & 0,229 & $<0,001$ \\
\hline \multicolumn{10}{|l|}{ Persönlichkeitsfaktoren } \\
\hline BFI-103: Extraversion $(n=721)$ & & & & & & & 0,132 & 0,096 & 0,172 \\
\hline BFI-103: Verträglichkeit $(n=721)$ & & & & & & & 0,058 & 0,116 & 0,617 \\
\hline BFI-103: Gewissenhaftigkeit $(n=721)$ & & & & & & & 0,292 & 0,116 & 0,012 \\
\hline BFI-103: Neurotizismus $(n=721)$ & & & & & & & $-0,309$ & 0,112 & 0,006 \\
\hline BFI-103: Offenheit $(n=721)$ & & & & & & & 0,146 & 0,092 & 0,114 \\
\hline $\begin{array}{l}\text { LOT- } R^{4}: \text { Dispositioneller Optimismus } \\
(n=720)\end{array}$ & & & & & & & 0,736 & 0,260 & 0,005 \\
\hline $\begin{array}{l}\text { LOT- } R^{4}: \text { Dispositioneller Pessimismus } \\
(n=718)\end{array}$ & & & & & & & $-0,520$ & 0,262 & 0,048 \\
\hline $\begin{array}{l}\text { IE- } 4^{5} \text { : internale Kontrollüberzeugung } \\
(n=720)\end{array}$ & & & & & & & 0,679 & 0,312 & 0,030 \\
\hline $\begin{array}{l}\text { IE- } 4^{5} \text { : externale Kontrollüberzeugung } \\
(\mathrm{n}=720)\end{array}$ & & & & & & & $-0,361$ & 0,287 & 0,208 \\
\hline Grübelneigung ( $\mathrm{n}=718$ ) & & & & & & & $-0,853$ & 0,172 & $<0,001$ \\
\hline \multicolumn{10}{|c|}{$\begin{array}{l}{ }^{1} \text { Referenzkategorie: weiblich } \\
2 \text { Referenzkategorie: Maßnahmen genau richtig } \\
{ }^{3} \text { BFI-10: Big Five Inventory-10 } \\
{ }^{4} \text { LOT-R: Life Orientation Test } \\
{ }^{5} \text { IE-4: Internale-Externale-Kontrollüberzeugung-4 } \\
{ }^{6} \text { B: Regressionskoeffizient } \\
{ }^{7} \text { Standardfehler }\end{array}$} \\
\hline
\end{tabular}


bußen ( $p=0,031)$ und ein höherer Konsum nichtalkoholischer psychotroper Substanzen $(p<0,001)$ mit schlechteren Befindlichkeitswerten assoziiert. Der Einschluss der Persönlichkeitsmerkmale in Modell 3 ( $\triangleright$ Tab. 1 ) erhöht die erklärte Varianz der WHO-5-Summenwerte auf 41,7\% (korrigiert, Signifikanz des Gesamtmodells: $F[23,627]=21,2 ; p<0,001)$. Aus dem Bereich coronabezogener Einschätzungen resultieren in diesem Modell weiterhin signifikante Effekte für Angst um die Gesundheit nahestehender Personen ( $p=0,044)$, Belastung durch Ausgangsbeschränkungen $(p<0,001)$ und Konsum psychotroper Substanzen $(p<0,001)$, die mit schlechteren Befindlichkeitswerten einhergehen. Aus dem Bereich der Persönlichkeitsmerkmale weisen Personen mit hoher Gewissenhaftigkeit $(p=0,012)$, niedrigen Neurotizismuswerten $(p=0,006)$, hohem Optimismus $(p=0,005)$, niedrigem Pessimismus $(p=0,048)$ hoher internaler Kontrollüberzeugung $(p=0,030)$ sowie geringer Grübelneigung $(p<0,001)$ bessere aktuelle Befindlichkeitswerte auf.

\section{Vergleich der psychischen Symptomatik der COVID-19-Umfrage 2020 mit der Umfrage 2018}

Quantitative Symptomausprägungen. Bezüglich der psychischen Befindlichkeitswerte war der WHO-5-Summenwert in den Umfragen 2018 und 2020 vergleichbar, nach Kontrolle von Alter, Geschlecht und Bildungsniveau unterschieden sich beide Stichproben nicht signifikant (2018 ( $n=423)$ : $M=56,83$ [95\%-KI 54,72;58,96]; $2020(n=716): 56,52$ [54,69; 58,11], $F=0,001$ [1100]; $p=0,992)$.

- Abb.2a (Onlineversion) zeigt die jeweiligen Summenwerte der quantitativen PHQ-D-Skalen für somatoforme, depressive, Angst- und Stresssymptome. In allen Skalen bestehen betragsmäßig kleine Gruppenunterschiede zuungunsten der 2020er-Umfrage. Die multivariate Kovarianzanalyse unter Einschluss der 4 Symptomskalen als abhängige Variablen und Kontrolle der soziodemografischen Variablen lieferte jedoch einen nichtsignifikanten Globaltest (Pillai-Spur, F[4,1053] = 1,15; $\mathrm{p}=0,330)$.

Anteil psychischer Beeinträchtigungen. \Online-Abb.2b zeigt für beide Umfragen die Anteile von Personen mit auffälligem WHO-5-Summenwert (<50, 2018: 34,5\% [30,0; 39,5]; 2020: 36,7\% [33,2; 40,4]) sowie die Anteile von Personen, die die Kriterien mindestens einer PHQ-D-Syndrom-Diagnose (PHQ-D psychische Störung, 2018: 26,8\% [22,6; 31,0], 2020: $29,4 \%[26,1 ; 33,0])$ und die Kriterien für mehrere PHQ-D-Diagnosen (PHQ-D-Komorbiditätsdiagnose, 2018: 8,9\% [6,2; 11,9], 2020: 10,2\% [7,9; 12,5]) erfüllten. Auch hier zeigen sich durchgängig betragsmäßig geringfügig ungünstigere Werte für die 2020er-Umfrage.

Unter Kontrolle der soziodemografischen Variablen im Rahmen logistischer Regressionen resultierten keine signifikanten Gruppenunterschiede in diesen Outcomes (WHO- $<50$ : Wald =0,379 (1); $\mathrm{p}=0$,538; PHQ-D psychische Störung: Wald = $1,03$ [1]; $P=0,311)$; PHQ-D-Komorbiditätsdiagnose: Wald $=$ $0,525[1] ; p=0,469)$.

\section{Diskussion}

\section{Coronabezogene Belastungen und Verhaltensweisen}

Unsere COVID-19-Umfrage zeigt eine breite Streuung wahrgenommener Belastungen in der Bevölkerung. Am häufigsten sind Ängste um die Gesundheit nahestehender Personen, während Ängste um die eigene Gesundheit deutlich seltener berichtet werden. Weitere häufige Belastungen betreffen Auswirkungen auf die familiäre Situation und Belastung durch Ausgangsbeschränkungen, gefolgt von Sorgen um den Arbeitsplatz bzw. um finanzielle Einbußen. Knapp 20\% der Befragten gibt an, zumindest teilweise mehr Alkohol zu konsumieren, verstärkter Konsum anderer psychotroper Substanzen wie Rauchen oder Medikamente wird von knapp 9\% bejaht. Inwieweit es sich hierbei um temporäre oder stabile Veränderungen handelt, lässt sich noch nicht abschätzen.

\section{Zusammenhänge von coronabezogenen Einschätzungen und Persönlichkeitsaspekten mit dem psychischen Befinden}

Unsere Analysen (Modell 1) identifizierten ein besseres psychisches Befinden bei älteren Personen und bei Personen mit höherem Bildungsniveau. Dies entspricht den Befunden epidemiologischer Feldstudien [19], aber auch anderer Bevölkerungsstudien im Kontext der Corona-Krise [6] und verweist auf generelle Protektivfaktoren, die auch während der COVID-19Pandemie wirksam sind. Dagegen war das psychische Befinden von Frauen und Männern in unserer Studie vergleichbar. Während eine Mehrzahl der Studien in Videgaard u. Benros [6] höhere psychische Beeinträchtigung bei Frauen fanden, wird dies nicht durchgängig bestätigt. Für fehlende Geschlechtsunterschiede wird u.a. angeführt, dass Männer ein generell höheres COVID-19-Erkrankungsrisiko und ein erhöhtes Risiko für maligne Krankheitsverläufe aufweisen, was zu einer Annäherung des psychischen Befindens von Männern und Frauen im Kontext der Pandemie führen mag [20]. Hier sind jedoch weitere Studien, auch zu möglichen Geschlechtsunterschieden im längerfristigen Verlauf der Pandemie, notwendig.

Unter Berücksichtigung dieser soziodemografischen Merkmale sind coronabezogene Einschätzungen aus verschiedenen Alltagsbereichen signifikant mit dem psychischen Befinden assoziiert (Modell 2). Subjektiv erlebte gute Informiertheit über das Virus geht mit besserem Befinden einher, dieser Zusammenhang findet sich bereits in anderen Arbeiten [6]. Ängste um die eigene Gesundheit und die Gesundheit nahestehender Personen, Belastungen durch Alltagsbeschränkungen und Sorgen um den Arbeitsplatz und/oder finanzielle Einbußen sind weitere Merkmale, die unabhängig voneinander mit schlechteren Befindlichkeitswerten einhergehen. Zudem konsumieren Personen mit schlechterem psychischen Befinden häufiger psychotrope Substanzen wie Nikotin und Medikamente.

Der Einbezug persönlichkeitsbezogener Risiko- und Resilienzfaktoren (Modell 3) führte zu einer deutlichen Verbesserung der Vorhersage der WHO-5-Summenwerte.

Als Persönlichkeitsfaktoren des BFI-10 sind niedrige Neurotizismuswerte und hohe Gewissenhaftigkeit mit besserem psy- 
chischen Befinden assoziiert. Diese Merkmale sind nach einer Metaanalyse mit 462 Studien eng mit psychischem Wohlbefinden und Lebenszufriedenheit assoziiert [15]. Als weitere förderliche Persönlichkeitsmerkmale resultierten hoher Optimismus, niedriger Pessimismus und hohe internale Kontrollüberzeugungen. Auch für diese Merkmale wurden in zahlreichen Längsschnittstudien positive Zusammenhänge mit psychischem Wohlbefinden identifiziert. Schließlich berichten Personen mit ausgeprägter Grübelneigung (Rumination) eine schlechtere psychische Befindlichkeit. Rumination stellt einen transdiagnostischen Risikofaktor für die Entstehung und den Verlauf einer Vielzahl von psychischen Störungen dar, insbesondere auch von Depressionen [21]. Bemerkenswert ist, dass all die hier untersuchten Persönlichkeitsmerkmale im statistischen Modell signifikante unabhängige Beiträge zur Vorhersage des aktuellen psychischen Befindens leisten. Jedoch gehen auch unter Berücksichtigung dieser Merkmale Ängste um die Gesundheit nahestehender Personen, wahrgenommene Belastungen durch Ausgangsbeschränkungen und vermehrter Substanzkonsum während der Krise weiterhin mit schlechterem psychischen Befinden einher.

\section{Vergleich mit der Bevölkerungsumfrage 2018}

Die beiden Bevölkerungsumfragen 2018 und 2020 wurden mit identischem Studiendesign realisiert. Dies ermöglichte den direkten Vergleich des psychischen Befindens anhand quantitativer Symptomskalen und prozentualer Anteile relevanter psychischer Beeinträchtigungen, die 2018 und 2020 mit denselben standardisierten Selbstbeurteilungsskalen (WHO-5, PHQD) erfasst wurden.

Bezüglich des WHO-5-Summenwerts als Globalmaß der psychischen Befindlichkeit resultierten keine signifikanten Unterschiede zwischen den Surveys. Mit Werten zwischen 56 und 57 Punkten liegen beide Surveys jedoch deutlich unter den aus der dänischen Studie ermittelten Werten (>60; [8]). Auch gegenüber dem European Quality of Life Survey 2016 [22] mit einem Wert von 65 für Deutschland wurden für beide Surveys schlechtere Werte ermittelt. Möglicherweise spielen hier zumindest teilweise regionale Besonderheiten (bzgl. Sozialstruktur, Arbeitslosigkeit etc.) eine Rolle. Für die quantitativen Syndromskalen des PHQ-D (Depression, Angst, somatoforme Beschwerden, Stress) wies ein Globaltest ebenfalls keine signifikanten Gruppenunterschiede nach.

Bei den prozentualen Anteilen psychischer Auffälligkeiten (WHO-5<50, Vorliegen mindestens einer psychischen Syndromdiagnose oder einer Komorbiditätsdiagnose nach PHQ-D) fällt auf, dass die entsprechenden Prozentsätze in der Umfrage 2020 um ca. 2 Prozentpunkte höher liegen als 2018, diese Unterschiede sind jedoch statistisch nicht signifikant.

Nach diesen Ergebnissen kann die in Medienberichten beschriebene Annahme einer dramatischen Zunahme psychischer Beeinträchtigungen nicht bestätigt werden. Zwischen den Umfragen 2018 und April/Mai 2020 fand sich keine signifikante Veränderung. Die vorliegende Studie stützt damit Empfehlungen, entsprechende Aussagen aus der rapide anwachsenden Zahl von Online-Studien mit fehlender Repräsentativität, unklaren Response-Raten und fehlenden Vergleichswerten aus Prä-
Pandemiezeiten an derselben Studienpopulation zumindest mit Vorsicht zu betrachten [11].

Wünschenswert sind nun repräsentative bundesweite und internationale Vergleichsstudien, die Aufschluss darüber geben, inwieweit regionale bzw. länderbezogene Unterschiede in der psychischen Gesundheit im Kontext der COVID-19-Pandemie und deren Entwicklung im weiteren Verlauf der Krise zu erwarten sind. Ein wichtiges Instrument stellen hier repräsentative Panel-Befragungen dar, die neben Vergleichen aus Präpandemiezeiten den Verlauf des Infektionsgeschehens, die Entwicklung der wirtschaftlichen und sozialen Situation sowie öffentliche Maßnahmen und Reaktionen darauf im Längsschnitt verfolgen und in Beziehung mit dem Verlauf der psychischen Gesundheit in der Bevölkerung setzen können. Eine solche Langzeitbefragung stellt z.B. das sozioökonomische Panel (SOEP) am Deutschen Institut für Wirtschaftsforschung (DIW Berlin) dar, dessen erste Ergebnisse zur Corona-Krise ähnlich wie unsere Studie zeigen, dass Indikatoren für psychische Belastungen (Lebenszufriedenheit, emotionales Wohlbefinden, Depressions- und Angstsymptome) bislang unverändert blieben (Stand: 3.6.2020) [23]. Eine längsschnittliche Untersuchung psychischer Belastungen im Kontext der COVID-19Pandemie ist insbesondere deshalb wichtig, da die Entwicklung der Krise und ihre wirtschaftlichen und sozialen Folgen (z.B. wiederholter Anstieg der Infektionsraten mit erneuten Lockdowns, Zunahme von Arbeitslosigkeit und finanzieller Unsicherheit) durchaus mittel- bis langfristige Effekte auf die psychische Gesundheit haben können, die momentan noch nicht abgebildet werden.

\section{Limitationen}

Die untersuchte Stichprobe ist nicht repräsentativ für die deutsche Bevölkerung, sondern beschränkt sich auf Einwohner aus Mannheim, einer mittelgroßen südwestdeutschen Stadt. Das Einschlusskriterium deutscher Staatsbürgerschaft wurde wegen der Sprachkenntnisse gewählt. Bei einem Migrantenanteil in Mannheim von insgesamt 45,3\% (davon 43,0\% mit deutscher Staatsangehörigkeit) kann deshalb über Migranten ohne deutsche Staatsangehörigkeit (25,8\% der Mannheimer Bevölkerung) keine Aussage gemacht werden. Aufgrund der Restriktion des Altersranges auf 18-65 können zudem keine Aussagen zu Kindern und Jugendlichen sowie älteren Menschen getroffen werden. Beide Gruppen könnten jedoch insbesondere aufgrund der Einschränkungen in den sozialen Kontakten besonders betroffen sein, Ältere auch durch ein höheres Erkrankungsrisiko. Zudem wurde nicht erfasst, ob die Befragten allein lebten. Auch für diese Personengruppe könnte die Corona-Krise eine besondere Belastung darstellen. Die Responserate der COVID19-Umfrage ist mit 36,9\% für einen schriftlichen sozialwissenschaftlichen Survey zufriedenstellend [24]. Dennoch kann ein systematischer Bias nicht ausgeschlossen werden, z. B. durch höhere Teilnahmebereitschaft von Personen, die sich durch die COVID-19-Krise stärker belastet fühlen. Inwieweit die mediale Darstellung der psychischen Folgen der COVID-19-Pandemie das Ausmaß der angegebenen Belastungen mitbeeinflusste, ist in diesem Kontext ebenfalls nicht beurteilbar. Zu den Angaben zu substanzbezogenem Verhalten kann die Unter- 
suchung schließlich nicht klären, inwiefern es sich hierbei um verstärktes vorbestehendes Problemverhalten handelt bzw. in welchem Ausmaß von Neuinzidenzraten problematischen Konsums auszugehen ist.

Über besonders gefährdete Gruppen wie Personen mit vorbestehenden psychischen Erkrankungen und Substanzgebrauchsstörungen kann die vorliegende Studie keine spezifischen Aussagen machen. Diese Menschen erfahren durch die Pandemie und die Einschränkungsmaßnahmen besonders weitreichende negative Konsequenzen [25, 26], die das Risiko von Symptomexazerbationen erhöhen. Auch zur Frage der diskutierten Zunahme des Suizidrisikos in der Bevölkerung aufgrund ökonomischer Verschlechterungen und sozialer Distanzierung [27] kann unsere Studie keinen Beitrag leisten.

Bei den eingesetzten Instrumenten zur psychischen Symptomatik handelt es sich um Selbstbeurteilungsfragebögen, die zwar sehr gute diagnostische Validitätskennwerte aufweisen $[9,10,18]$, jedoch keine klinischen Diagnosen ermöglichen. Schließlich erlaubt der Querschnittscharakter unserer Studie keine kausalen Aussagen zu Konsequenzen der COVID-19-Pandemie auf die psychische Gesundheit, sie kann nur Zusammenhänge beschreiben.

\section{KONSEQUENZEN FÜR KLINIK UND PRAXIS}

- Wahrgenommene Belastungen und Verhaltensweisen (hier: Substanzkonsum) während der COVID-19-Pandemie gehen mit schlechterem psychischen Befinden einher.

- Große Bedeutung für das psychische Befinden während der Krise haben individuelle Risiko- und Resilienzfaktoren. Neben der Ausweitung von Hilfsangeboten für Menschen mit psychischen Störungen $[25,26]$ sind deshalb Initiativen zur Vermittlung von Strategien zur Belastungsreduktion und Resilienzförderung zu begrüßen, die derzeit von relevanten Fachgesellschaften für eine breitere Öffentlichkeit angeboten werden (z. B. [28]).

- Zwischen 2018 und 2020 resultierten keine deutlichen Veränderungen von Indikatoren psychischer Störungen. Hier sind längsschnittliche Erhebungen zu mittel- und längerfristigen Auswirkungen wünschenswert.

Interessenkonflikt

Die Autorinnen/Autoren geben an, dass kein Interessenkonflikt besteht.

\section{Literatur}

[1] Johns Hopkins University and Medical Center. COVID-19 Dashboard by the Center for Systems Science and Engineering (CSSE) at Johns Hopkins University (JHU). 2020: Available from: https://coronavirus. jhu.edu/map.html
[2] Brooks SK, Webster RK, Smith LE et al. The Psychological Impact of Quarantine and How to Reduce It: Rapid Review of the Evidence. Lancet 2020; 395: 912-920

[3] Röhr S, Müller F, Jung F et al. Psychosoziale Folgen von Quarantänemaßnahmen bei schwerwiegenden Coronavirus-Ausbrüchen: ein Rapid Review. Psychiat Prax 2020; 47: 179-189

[4] Bohlken J, Schomig F, Lemke MR et al. COVID-19-Pandemie: Belastungen des medizinischen Personals. Psychiat Prax 2020; 47: 190197

[5] Pappa S, Ntella V, Giannakas T et al. Prevalence of Depression, Anxiety, and Insomnia Among Healthcare Workers During the COVID-19 Pandemic: A Systematic Review and Meta-Analysis. Brain Behav Immun 2020. doi:10.1016/j.bbi.2020.05.026

[6] Vindegaard N, Eriksen Benros M. COVID-19 Pandemic and Mental Health Consequences: Systematic Review of the Current Evidence. Brain Behav Immun 2020. doi:10.1016/j.bbi.2020.05.048

[7] Blake H, Bermingham F, Johnson G et al. Mitigating the Psychological Impact of COVID-19 on Healthcare Workers: A Digital Learning Package. Int J Environ Res Public Health 2020; 17: 2997. doi:10.3390/ ijerph17092997

[8] Sønderskov KM, Dinesen PT, Santini Zl et al. The Depressive State of Denmark During the COVID-19 Pandemic. Acta Neuropsychiatr 2020. doi:10.1017/neu.2020.15

[9] Krieger T, Zimmermann J, Huffziger $S$ et al. Measuring Depression With a Well-Being Index: Further Evidence for the Validity of the WHO Well-Being Index (WHO-5) as a Measure of the Severity of Depression. J Affect Disord 2014; 156: 240-244

[10] Topp CW, Ostergaard SD, Sondergaard S et al. The WHO-5 Well-Being Index: A Systematic Review of the Literature. Psychother Psychosom 2015; 84: 167-176

[11] Pierce M, McManus S, Jessop C et al. Says Who? The Significance of Sampling in Mental Health Surveys During COVID-19 Lancet Psychiatry 2020. doi:10.1016/ S2215-0366(20)30237-6

[12] Kovaleva A, Beierlein C, Kemper C] et al. Eine Kurzskala zur Messung von Kontrollüberzeugung: Die Skala Internale-Externale-Kontrollüberzeugung-4 (IE-4). (GESIS-Working Papers, 2012/19). Mannheim: GESIS - Leibniz-Institut für Sozialwissenschaften; 2012: Available from https://nbn-resolving.org/urn:nbn:de:0168-ssoar-312096

[13] Rammstedt B, Kemper C], Klein MC et al. Eine kurze Skala zur Messung der fünf Dimensionen der Persönlichkeit: Big-Five-Inventory-10 (BFI10). (GESIS-Working Papers, 2012/23). Mannheim: GESIS - LeibnizInstitut für Sozialwissenschaften; 2012: Available from: https://nbnresolving.org/urn:nbn:de:0168-ssoar-312133

[14] Glaesmer H, Rief W, Martin A et al. Psychometric properties and population-based norms of the Life Orientation Test Revised (LOT-R). Br J Health Psychol 2012; 17: 432-445. doi:10.1111/j.2044-8287.2011. 02046.x

[15] Anglim J, Horwood S, Smillie LD et al. Predicting Psychological and Subjective Well-Being from Personality: A Meta-Analysis. Psychol Bull 2020; 146: 279-323

[16] Dreßing H, Gass P, Schultz K et al. The Prevalence and Effects of Stalking - A Replication Study. Dtsch Ärztebl Int 2020; 117: 343-353. doi:10.3238/ärztebl.2020.0347

[17] Beddig T, Kuehner C. Ambulatory Assessment Characteristics Predict the Clinical Course of Premenstrual Dysphoric Disorder. Psychother Psychosom 2020. doi:10.1159/000505999

[18] Löwe B, Spitzer RJ, Zipfel S, Herzog W. Gesundheitsfragebbogen für Patienten (PHQ-D). Komplettversion und Kurzform. Karlsruhe: Pfizer; 2002

[19] Jacobi F, Höfler M, Strehle J et al. Twelve-Months Prevalence of Mental Disorders in the German Health Interview and Examination Survey for Adults - Mental Health Module (DEGS1-MH): A Methodological Addendum and Correction. Int J Methods Psychiatr Res 2015; 24: 305313 
[20] Gao ], Zheng P, Jia Y et al. Mental Health Problems and Social Media Exposure During COVID-19 Outbreak. PLoS One 2020; 15: e0231924. doi:10.1371/journal.pone.0231924

[21] Kuehner C. Why is Depression More Common Among Women Than Among Men? Lancet Psychiatry 2017; 4: 146-158

[22] European Foundation for the Improvement of Living and Working Conditions. European Quality of Life Survey 2016. Available from: https://www.eurofound.europa.eu/data/european-quality-of-lifesurvey

[23] Entringer T, Kröger H. Einsam, aber resilient - Die Menschen haben den Lockdown besser verkraftet als vermutet. DIW aktuell 2020: 46. https://www.diw.de/documents/publikationen/73/diw_01. c.791373.de/diw_aktuell 46.pdf

[24] de Leeuw E, Hox J, Luiten A. International Nonresponse Trends Across Countries and Years: An Analysis of 36 Years of Labour Force Survey
Data. Survey Insights: Methods From the Field 2018. Available from www.surveyinsights.org/?p=10452

[25] Frank A, Hörmann S, Krombach J et al. Psychisch krank in Krisenzeiten: Subjektive Belastungen durch COVID-19. Psychiat Prax 2020; 47: 267-272. doi:10.1055/a-1179-4230

[26] Richter D, Zürcher S. Psychiatrische Versorgung während der COVID19-Pandemie. Psychiat Prax 2020; 47: 173-175. doi:10.1055/a-11578508

[27] Reger MA, Stanley IH, Joiner TE. Suicide Mortality and Coronavirus Disease 2019 - A Perfect Storm? JAMA Psychiatry 2020. doi:10.1001/ jamapsychiatry.2020.1060

[28] Deutsche Gesellschaft für Psychologie (DGPs). Psychologische Hilfe während der Corona-Krise 2020. Available from: https://psychologische-coronahilfe.de 